\title{
Early black-hole seeds in the first billion years
}

\author{
Umberto Maio* \\ INAF - Italian National Institute for Astrophysics, via A. Corti 12, 20133 Milano (Italy) \\ E-mail: umberto.maio a inaf.it
}

Supermassive black holes with billion solar masses are in place already within the first Gyr, however, their origin and growth in such a short lapse of time is extremely challenging to understand. Here, we discuss the formation paths of early black-hole seeds, showing the limits of light blackhole seeds from stellar origin and the expected characteristics of heavy/massive black-hole seeds originated by gas direct collapse in peculiar primordial conditions. To draw conclusions on the possible candidates and the role of the ambient medium, we use results from N-body hydrodynamic simulations including atomic and molecular non-equilibrium abundance calculations, cooling, star formation, feedback mechanisms, stellar evolution, metal spreading of several heavy elements from SNII, AGB and SNIa, and multifrequency radiative transfer over 150 frequencies coupled to chemistry and SED emission for popII-I and popIII stellar sources. Standard stellar-origin light black holes are unlikely to be reliable seeds of early supermassive black holes, because, under realistic assumptions, they cannot grow significantly in less than a billion years. Alternatively, massive black-hole seeds might originate from direct collapse of pristine gas in primordial quiescent mini-haloes that are exposed to stellar radiation from nearby star forming regions. The necessary conditions required to form heavy seeds must be complemented with information on the complex features of local environments and the fine balance between chemistry evolution and radiative transfer.

Multifrequency Behaviour of High Energy Cosmic Sources - XIII - MULTIF2019

3-8 June 2019

Palermo, Italy

${ }^{*}$ Corresponding author. 


\section{Introduction}

Black holes (BHs) with billion solar masses are observed up to redshifts $z \simeq 7.5$, when the Universe was only about 0.7 Gyr old [Fan et al.(2001), Mortlock et al.(2011), Bañados et al.(2018)]. These evidences make the birth of the first supermassive black holes (SMBHs) one of the most striking events in the first billion years. However, how such large objects could form and grow in such a short lapse of time is extremely challenging to understand. The role of the environment in which these extreme objects appear is an unknown issue, as well as the role of the first primordial stellar populations injecting UV photons in the early intergalactic medium (IGM). In fact, it is still debated whether the origin of SMBHs should be linked to 'light' stellar-origin BH seeds (with masses $\sim 1-10^{2} \mathrm{M}_{\odot}$ ) or to 'heavier' massive $\mathrm{BH}$ seeds (with masses $\sim 10^{4}-10^{6} \mathrm{M}_{\odot}$ ) [Rees(1984)]. Furthermore, it is difficult to predict under which physical conditions these seeds could be born, how they relate to nearby structure formation episodes and what the interplay with the ongoing feedback processes is.

The first baryonic structures are supposed to be born in small primordial dark-matter haloes hosting molecular evolution, star formation and metal pollution. Primordial haloes are expected to be $\mathrm{H}_{2}$-rich, hence the pristine gas they host can cool and fragment, producing the first population III (popIII) stars, the first stellar black holes and the first heavy elements in cosmic history. Metal spreading is then responsible for increasing the cooling capabilities of nearby regions [Maio et al.(2007)], while the emitted UV photons can ionise the IGM totally or partially depending on stellar masses and explosion energies. Given that stellar populations described by different initial mass functions (IMFs) produce different amounts of BH remnants, metal spreading and ionizing photons, the actual IMF in star forming regions at different epochs influence significantly BH formation and growth. Indeed, stellar populations described by conventional Salpeter-like IMFs will produce stellar BHs mostly in the mass range $1-10 \mathrm{M}_{\odot}$ after a few $10^{7} \mathrm{yr}$ from their formation time. Top-heavy IMFs, instead, will lead to the formation of more massive stars and BHs with masses of the order of $\sim 10^{2} \mathrm{M}_{\odot}$. Metal enrichment is also going to change drastically depending on the original IMF, with typical yields ranging from a few per cent in the Salpeter-like case up to $50 \%$ in the top-heavy case. Furthermore, the emitted photons will create a background radiation field that might ionise and/or dissociate $\mathrm{H}_{2}$ molecules around star forming regions. The strength of radiation as well as the shape and extension of the impacted regions will depend on the stellar mass range expected by different IMFs. This is particularly important when addressing the effects of stellar evolution on the chemical and thermal conditions of the cosmic medium. Indeed, pristine gas in early mini-haloes illuminated by UV radiation might keep an atomic state around $10^{4} \mathrm{~K}$, be prevented from cooling and eventually collapse directly into a massive $\mathrm{BH}$, forming a so-called direct-collapse BH (DCBH).

To shed light on the seeds of SMBHs, it is crucial to study thermal, chemical and radiative properties of cosmic structures during cosmological epochs. For a complete picture, it is necessary to follow gravity and hydrodynamics coupled to molecule formation and metal production from stellar evolution. Indeed, (i) molecules lead the first gas collapsing events in primordial pristine gas; (ii) metals, spread out by the first stars, increase gas cooling capabilities with their large variety of atomic transitions influencing subsequent structure formation; (iii) stellar evolution determines typical timescales, metal yields, photon production and BH masses. Given the high non-linearity 
of these processes, to address adequately the problem, one has to implement atomic and molecular cooling, star formation, metal enrichment from type II and type Ia supernovae (SNe), as well as AGB phases and radiative transfer (RT) in dedicated N-body hydrodynamic numerical simulations of the first Gyr.

As an example, in Fig. 1, simulated $\mathrm{H}_{2}$-driven gas collapse and inflow from a zoom into a primordial star forming halo, as expected by numerical simulations [Maio and Tescari(2015)], is displayed. The colour code corresponds to different values of gas overdensity, $\delta$, during a time span of roughly $1.4 \mathrm{Gyr}$. In this period, $\delta$ increases from values around unity or less up to $\delta>10^{3}$ (red spot in the rightmost panel), where most of the gas mass results concentrated. In typical situations, the overdense gas clump just formed is going to cool, fragment and form stars. This is highlighted by Fig. 2 which shows how, simultaneously in the same time span, early gas clumps host first episodes of star formation accompanied by shock heating and metal enrichment in the neighbouring regions. Highly non-linear effects related to the underlying structure evolution (e.g. development of knots and filaments) are evident. In these chaotic environments, where star formation, feedback and photon production coexist, the first BHs, seeds of the observed SMBHs, can be born.

In the next, we will focus on the possible channels by which early BH seeds form and on the effects from the local environment. The work is organized as follows: in Sect. 2, we present the main features of light BH seeds, in Sect. 3, we show the path leading to the formation of heavy BH seeds, while in Sect. 4, we summarise and conclude.
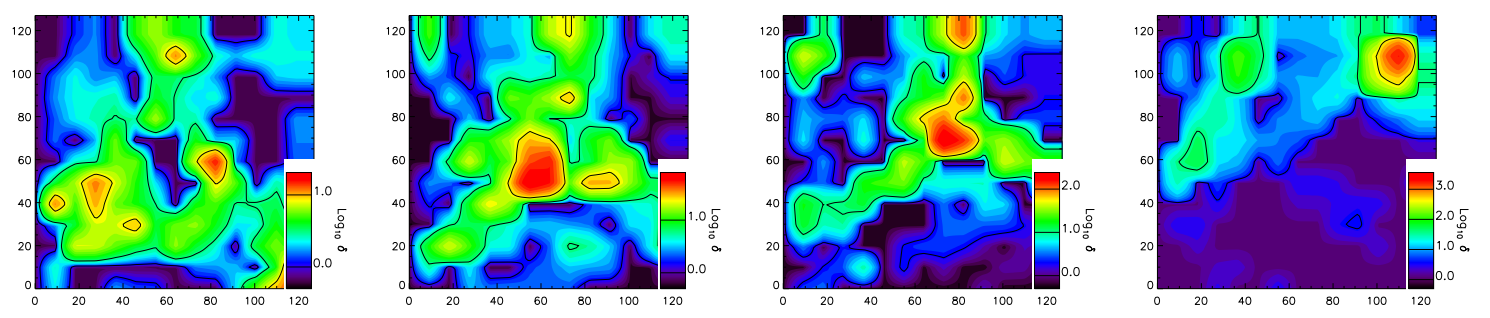

Figure 1: Simulated $\mathrm{H}_{2}$-driven gas collapse and inflow of a primordial star forming halo displayed through the projection of gas overdensity, $\delta$, on a $128 \times 128$ pixel grid. The region corresponds to $600 \mathrm{kpc}$ (comoving) at redshift $z \simeq 7$ and the following $0.4,0.8$ and $1.4 \mathrm{Gyr}$, respectively, from left to right.
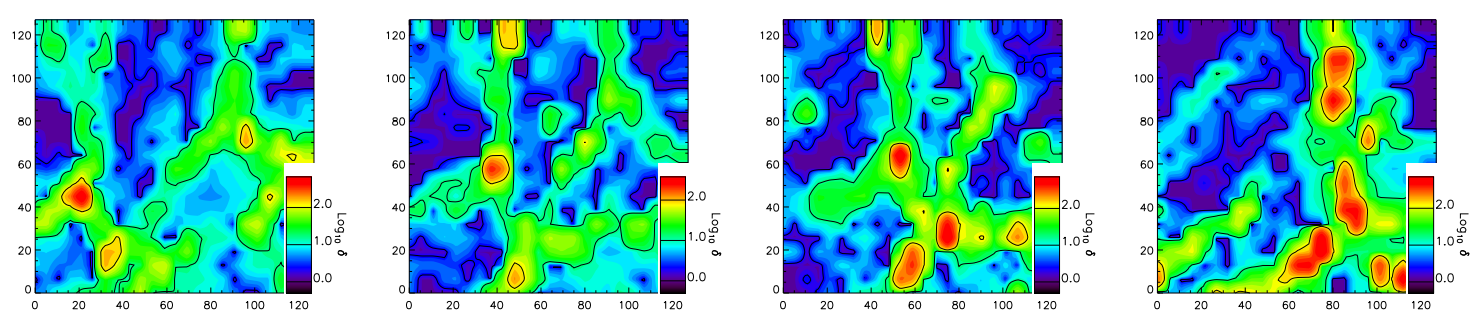

Figure 2: Simulated star formation and stellar-origin BH generation in a primordial star forming halo displayed through the projection of gas overdensity, $\delta$, on a $128 \times 128$ pixel grid. The region corresponds to $1000 \mathrm{kpc}$ (comoving) at $z \simeq 7$ over 1.4 Gyr time evolution, as in Fig. 1. 


\section{Light seeds: stellar black holes}

Light black-hole seeds are originated at the end of the life of stars. In early regimes, cosmic gas has pristine or very metal-poor chemical composition and is able to host popIII star formation. The typical properties of the popIII regime, such as stellar IMF, explosion energies or BH remnant masses, are still largely unknown. Theoretical predictions for primordial massive stars suggest that they should produce significant amounts of heavy elements that quickly pollute nearby gas and mark the transition to the standard population II-I (popII-I) regime [Maio et al.(2010)].

The popII-I regime takes place in enriched star formation sites (above a critical metallicity of $\sim 10^{-4} \mathrm{Z}_{\odot}$ ) and the expected mass distribution features a power-law shape (Salpeter IMF or similar). Standard stellar-evolution calculations suggest $\mathrm{SN}$ explosion energies around $\sim 10^{51} \mathrm{erg}$, which is not sufficient to drive large-scale reionization by $z \simeq 6$, but is enough to produce correct star formation rates (SFRs) and magnitudes [Dayal et al.(2013), Mancini et al.(2016)], as well as pollute the cosmic medium with metallicities [Salvaterra et al.(2013), Maio et al.(2013)], abundance ratios [Ma et al.(2017), Ma et al.(2017)] and dust masses [Mancini et al.(2015)] consistent with the observations. The interplay among feedback processes from different stellar populations might affect pollution of the cosmic medium, although details are tightly dependent on the assumptions. Both popIII and popII-I generations can produce BH remnants. In the former case, massive popIII stars can leave BHs with masses of the order of $\sim 10^{2} \mathrm{M}_{\odot}$, while in the latter case, one expects remnant $\mathrm{BH}$ masses around $\sim 1-10 \mathrm{M}_{\odot}$. This means that stellar-origin $\mathrm{BHs}$ are good candidates for light black-hole seeds at all times.

Since, the transition from popIII to popII-I generations is quite fast at redshift larger than $\sim 15$, the typical stellar-origin BH masses in the first Gyr are expected to be dominated by popII-I objects. This is highlighted by Fig. 3, where the formation rate of stellar-origin BHs is plotted, as derived from simulated SFR densities for popIII and popII generations. These numerical calculations are performed with an updated version of the N-body smoothed-particle hydrodynamics (SPH) code Gadget-3 [Springel(2005)] and the numerical implementation takes into account: primordial non-equilibrium chemistry, atomic and molecular cooling in the temperature regime $\sim 10-10^{9} \mathrm{~K}$ [Maio et al.(2007)], star formation, feedback effects and stellar evolution with metal spreading [Tornatore et al.(2007)] from SNII [Woosley and Weaver(1995)], AGB [van den Hoek and Groenewegen(1997)] and SNIa [Thielemann et al.(2003)] phases for both popIII and popII-I generations according to metal-dependent yields and a critical metallicity for popIII to popII-I transition of $Z_{\text {crit }}=10^{-4} \mathrm{Z}_{\odot}$. The IMF used for regular popII-I regimes is a Salpeter one over $[0.1,100] \mathrm{M}_{\odot}$ range, while the IMF used for popIII regimes is a top-heavy one over [100, 500] $\mathrm{M}_{\odot}$ range with the same slope as the Salpeter [Maio et al.(2010)]. Stars with masses lower than $\sim 8 \mathrm{M}_{\odot}$ evolve through $\mathrm{AGB}$ and SNIa phases, while larger stars evolve as SNII, leaving as remnant a neutron star (if the progenitor mass is lower than $\sim 40 \mathrm{M}_{\odot}$ ) or a $\mathrm{BH}$ (if the progenitor mass is in the range $\sim 40-100 \mathrm{M}_{\odot}$ ). ${ }^{1}$ In this latter case, the expected stellar lifetime is $\lesssim 30$ Myr. PopIII BHs are formed from the death of stars with masses in the ranges $100-140 \mathrm{M}_{\odot}$

\footnotetext{
${ }^{1}$ The threshold mass to distinguish the formation of neutron stars from BHs bears uncertainties with stellar models predicting values between $20 \mathrm{M}_{\odot}$ and $40 \mathrm{M}_{\odot}$ [Campisi et al.(2011), and references therein]. For a Salpeter IMF, the resulting difference on the $\mathrm{BH}$ mass fraction is less than a factor of 2 and ranging from 0.037 (with threshold mass of $40 \mathrm{M}_{\odot}$ ) to 0.074 (with threshold mass of $20 \mathrm{M}_{\odot}$ ).
} 


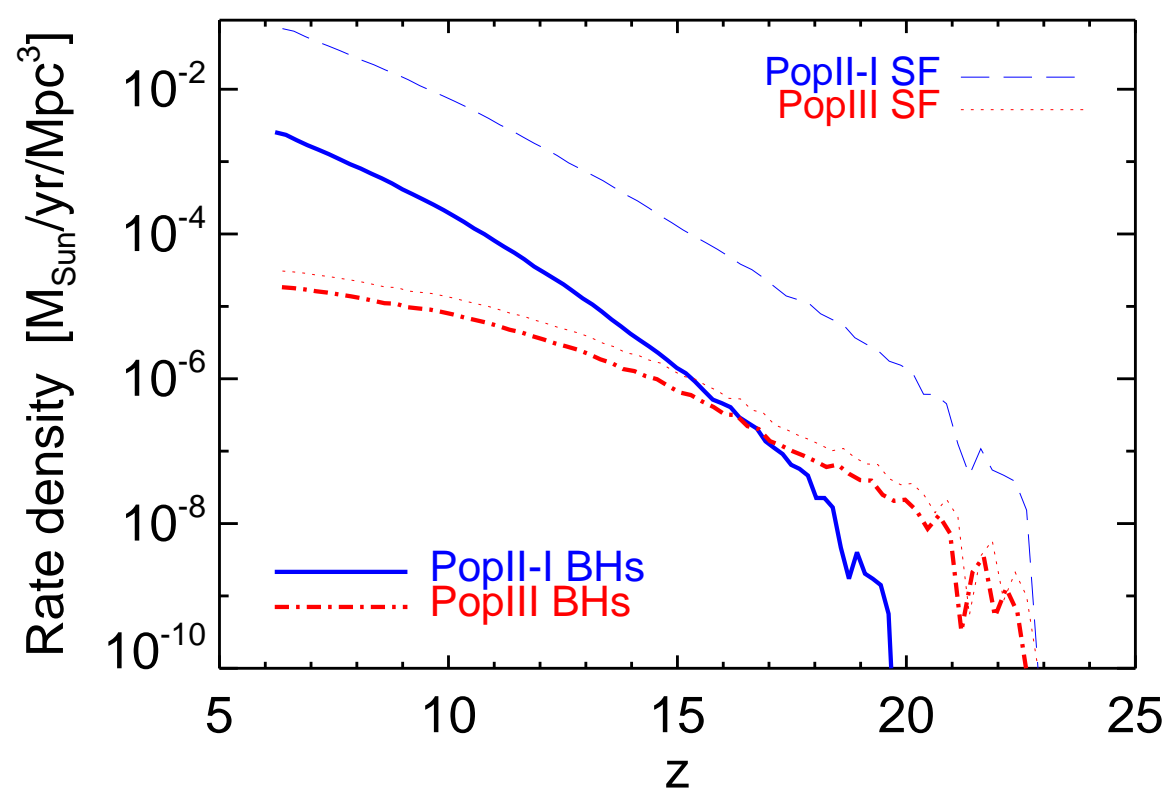

Figure 3: Stellar-origin BHs for different populations, as expected from the primordial cosmic star formation rate. The $\mathrm{BH}$ formation rate density for popII-I (solid line) and popIII (dash-dotted line) is plotted, as derived from simulated SFR densities for popII-I (dashed line) and popIII (dotted line) regimes.

and 260-500 $\mathrm{M}_{\odot}$ after a rapid evolution of roughly $2 \mathrm{Myr}$. Stars in the intermediate mass range of 140-260 $\mathrm{M}_{\odot}$ die as pair-instability SNe (PISNe) without leaving any remnant.

PopII-I SNe have explosion energies of $10^{51}$ erg [Woosley and Weaver(1995), ] and popIII PISNe produce mass-dependent energies between $10^{51}$ and $10^{53} \mathrm{erg}$ [Heger and Woosley(2002), ]. These events heat, pollute and inject entropy in the surrounding medium. The metals ejected during stellar evolution are diffused to the neighbouring regions via the SPH kernel, while kinetic feedback is modelled though wind velocities of $500 \mathrm{~km} / \mathrm{s}$.

In our specific case (Fig. 3), we adopt cosmological initial conditions at redshift 100 and evolve them down to redshift 6 , in a box with side of $10 \mathrm{Mpc} / h$ with expansion parameter normalised to $100 \mathrm{~km} / \mathrm{s} / \mathrm{Mpc} h=0.7$, present-day matter and $\Lambda$ density parameters $\Omega_{0, m}=0.27$ and $\Omega_{0, \Lambda}=0.73$, flat cosmology and initial number particles of $2 \times 512^{3}$ for gas and dark matter, respectively [Maio and Viel(2015), Maio and Tescari(2015), and references therein for more details]. The plot displays popII-I and popIII SFR densities at $z=5-25$ and the corresponding popII-I and popIII $\mathrm{BH}$ formation rate densities, computed including the appropriate time delays for each population. The trends of the SFR densities are consistent with the ones expected in literature and feature an increasing trend in time, during which popIII star formation (SF, dotted line) becomes more and more subdominant with respect to the popII-I SF (dashed line). Although at $z>20$ the two regimes are comparable, by redshift $z \simeq 10$ the discrepancy reaches 3 orders of magnitude and keeps increasing. The corresponding BH formation rates are dictated by the SFR. For the popIII BH formation rate density (dash-dotted line), there is a short time delay induced by the short lifetimes of very massive 
stars (2 Myr). On the contrary, for popII-I BHs (solid line), the time delay is larger (30 Myr) and this makes the curve steeper than the SFR, mostly at higher $z$, when the effect is more evident due to the non-linearity of the time-redshift relation. As a result, the solid line increases slightly after $z \simeq 20$ and then intersects the dash-dotted line around $z \simeq 16$. After this redshift, popII-I BHs are produced more abundantly than popIII BHs (by 1 dex around $z \simeq 10$ and 2 dex around $z \simeq 6$ ). The epochs before $z \simeq 16$, instead, feature a more significant contribution of popIII BHs ( 3 dex at $z \simeq 20$ ), as a consequence of their almost instantaneous link to popIII SFR.

In fact, popII-I BHs dominates at most times, since already the earliest popIII star formation episodes pollute the surrounding haloes above the critical level [Biffi and Maio(2013)] and the popIII contribution to the cosmic SFR density drops irrespectively of exact numerical parameters. This means that the residual fraction of $\sim 10^{2} \mathrm{M}_{\odot}$ BH remnants is tiny and available $\mathrm{BH}$ seeds lie mostly in the lower mass tail, i.e. $\sim 1-10 \mathrm{M}_{\odot}$. Unfortunately, these masses are too small to justify the existence of SMBHs at $z \simeq 7$. It must be noted that, according to the ordinate values, a hypothetical rapid merger of all the light BHs formed by $z \sim 6$ (in one Gyr) would produce about $10^{7} \mathrm{M}_{\odot} / \mathrm{Mpc}^{3}$. Studies investigating accretion processes onto stellar-origin BHs [Hirano et al.(2014)] find that, even in the most favourable conditions, BH seeds of the order of $\sim 10^{2} \mathrm{M}_{\odot}$ hardly grow in a few hundred Myr. These considerations imply the need to search for alternative ways to form heavier seed BHs that might grow into SMBHs by $z \simeq 7$ [Valiante et al.(2018), Natarajan et al.(2019), and references therein].

\section{Heavy seeds: direct-collapse black holes}

A possible alternative to form SMBHs at high redshift is represented by gas accretion on $\mathrm{BH}$ seeds with initial masses of $\sim 10^{4}-10^{6} \mathrm{M}_{\odot}$. Forming such heavy seeds is rather difficult, though. Indeed, dynamical interactions and coalescence of light BHs have long timescales to allow the formation of a single $10^{4}-10^{6} \mathrm{M}_{\odot}$ BH in less than a Gyr. On the other hand, the direct-collapse scenario, a rapid collapse of the gas in primordial haloes into massive BHs [Bromm and Loeb(2003), Begelman et al.(2006)], seems plausible in early epochs. DCBHs are expected to form in particular environments where pristine gas is not fragmenting nor forming stars. This requires that molecular cooling, effective at temperatures below $\sim 8 \times 10^{3} \mathrm{~K}$, should get dissociated by external $U V$ radiation in the Lyman-Werner (LW) band. In order to keep the gas bound in early minihaloes, one expects that the minimum dark-matter mass of the hosting candidate should be of the order of, or larger than, $\sim 2 \times 10^{6} \mathrm{M}_{\odot}$ to prevent photo-evaporation due to photo-heating and feedback mechanisms from nearby star forming regions. In fact, hosting candidates should be haloes with virial temperature around $10^{4} \mathrm{~K}$ and exposed to significative amounts of LW flux, $J_{\mathrm{LW}}$. The amount of photons emitted in the LW band depends on the adopted spectral properties of primordial sources [Sugimura et al.(2014)], however, literature studies have shown that dissociating LW fluxes (in units of $10^{-21} \mathrm{erg} / \mathrm{s} / \mathrm{cm}^{2} / \mathrm{Hz} / \mathrm{sr}$, hereafter $J_{21}$ ) are effective in the range of values $J_{\mathrm{LW}} \sim 1$ $1000 J_{21}$, while the effects are dramatic for much bigger values, preventing DCBH formation by the end of the first Gyr [Shang et al.(2010), Wolcott-Green et al.(2011), Habouzit et al.(2016), Johnson and Dijkstra(2017), etc.]. As an example, one might expect that the gas clump in the rightmost panel of Fig. 1, where the overdensity is $\delta>10^{3}$, under these peculiar conditions might be a good candidate for hosting DCBH formation at redshift $z \gtrsim 10$. 
In general, simplified numerical simulations are not capable to address the process of DCBH formation, because the dominant physical mechanism ruling DCBH formation is radiative feedback coupled to non-equilibrium chemistry [Petkova and Maio(2012)]. Furthermore, one has to take into account the different spectral energy distributions (SEDs) emitted by sources from different stellar populations to address photo-ionization and photo-heating of the gas in the haloes near star formation sites [Maio et al.(2016)]. Indeed, these latter haloes are the ones that might be exposed to sufficient ionizing radiation and be host candidates of DCBHs.

We implement the needed numerical schemes, including all the physical treatments mentioned in the previous section (primordial non-equilibrium chemistry, atomic and molecular cooling, feedback effects, star formation, stellar evolution and metal spreading from SNII, AGB and SNIa for popIII and popII-I generations, a critical metallicity of $Z_{\text {crit }}=10^{-4} \mathrm{Z}_{\odot}$ ) and, in addition, multifrequency RT according to the Eddington tensor formalism coupled to atomic and molecular nonequilibrium calculations for both popII-I and popIII stellar generations [Maio et al.(2019)]. The SEDs used are black bodies sampled in the same frequency range as the non-equilibrium chemical calculations (150 frequency bins), in order to compute chemical radiative rates consistently with radiative fluxes from active sources. For popII-I generations a Salpeter IMF and a popII-I SED approximated as $10^{4} \mathrm{~K}$ black-body emission are adopted. For popIII generations we consider two cases: a powerful case with a top-heavy IMF and popIII SED given by a $10^{5} \mathrm{~K}$ black-body emission; a weak case with popIII stars similar to popII-I, having a Salpeter IMF and a $10^{4} \mathrm{~K}$ black-body SED. We adopt initial conditions at redshift 100 and evolve them down to redshift 6 , in a box of 0.5 $\mathrm{Mpc} / h$ a side sampled by $2 \times 256^{3}$ for gas and dark matter, respectively, with $h=0.7, \Omega_{0, m}=0.3$ and $\Omega_{0, \Lambda}=0.7$. These choices are motivated by the need of a trade-off configuration in which physical, chemical and radiative processes are followed accurately while still resolving primordial structures [Maio et al.(2019), and references therein].

The effects of the different assumptions for IMFs and SEDs from popIII generations are shown in the maps of Fig. 4. DCBH host candidates are searched according to the above-mentioned requirements, however, only in the powerful case three haloes, located several physical kpc from the central popIII star forming region, fulfil the conditions (candidate A, B and C in the Figure). In the weaker case, there are no candidates, since the structures that are neither forming stars nor impacted by stellar feedback mechanisms typically host cold gas with temperatures below $10^{4} \mathrm{~K}$. The three candidates A, B and C have gas masses of $1-3 \times 10^{5} \mathrm{M}_{\odot}$ and are exposed to LW radiation of $J_{\mathrm{LW}} \sim 1-50 J_{21}$, which completely dissociates $\mathrm{H}_{2}$ molecules. These values for $J_{\mathrm{LW}}$ are consistent with the lower-end of the (rather large) range suggested by literature studies. The gas residing in these haloes shows turbulent patterns at all resolved scales with Reynolds numbers around $10^{4}-10^{8}$, consistently with what expected for early mini-haloes at these epochs [Maio et al.(2011)]. The distance of the candidates $\mathrm{A}, \mathrm{B}$ and $\mathrm{C}$ from the central source is larger than 5 physical kpc. This value should be considered as a lower limit for DCBH formation, since gas closer to the central star forming region is heavily affected by metal enrichment and stronger photo-evaporation due to the larger LW intensities. At distances $\gtrsim 5 \mathrm{kpc}$, material is not significantly polluted and photo-evaporation is milder as a consequence of the decease of radiation flux with distance. Since metal spreading inhibits DCBH formation near star forming regions, where $J_{\mathrm{LW}}$ is larger, one must conclude that this channel to form heavy seeds requires a very fine balance between chemical feedback and radiative feedback. Nevertheless, mechanical feedback due to local substructures or clump interactions 

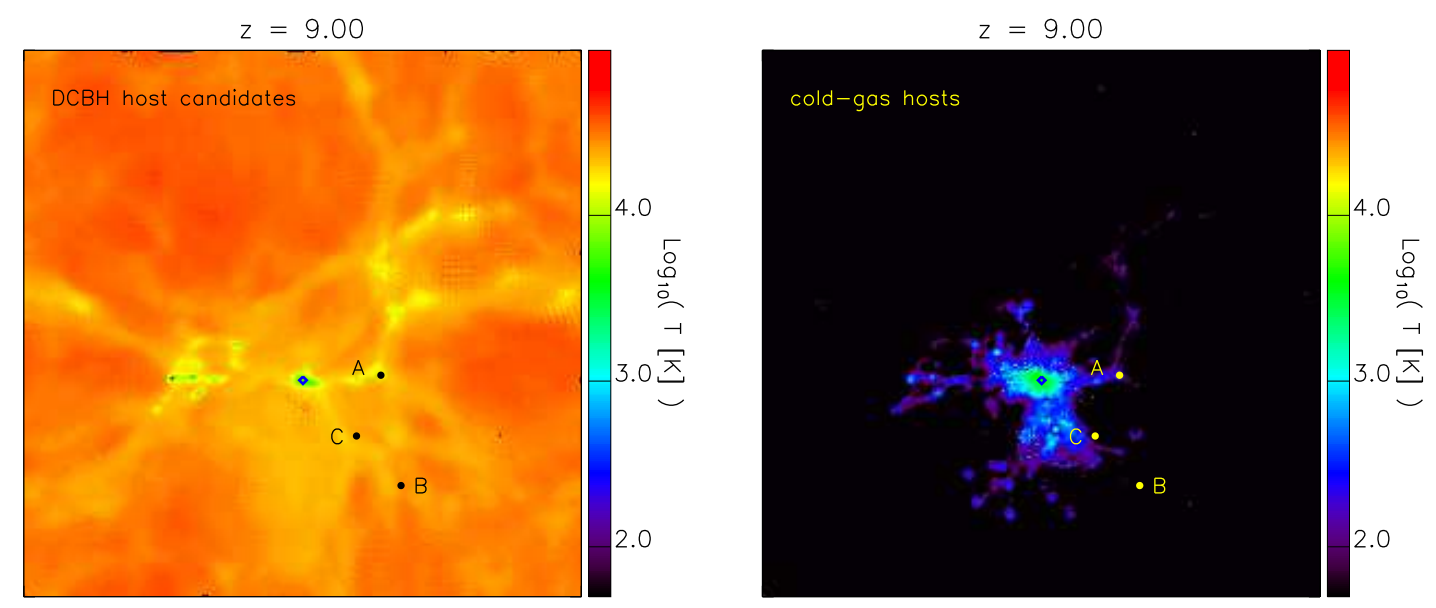

Figure 4: Temperature maps at $z=9$ for a run with a top-heavy IMF and a powerful SED corresponding to $10^{5} \mathrm{~K}$ black-body emission (left) and for a run with a Salpeter IMF and a weaker SED corresponding to $10^{4} \mathrm{~K}$ black-body emission (right). The black bullets on the left map highlight the positions of DCBH host candidates along cosmic filaments. The yellow bullets on the right map are the same objects, but in this case host cold gas. In both maps, the central star forming region is denoted by an empty diamond.

may further harm DCBH formation. A closer look to the three haloes reveals that candidate A is composed by one single roundish structure, candidate B by an irregular elongated shape where gas is interacting and candidate $\mathrm{C}$ by two distinct sub-clumps. Such local non-linear processes will influence the evolution of the latter two candidates at later times and possibly inhibit DCBH formation [Maio et al.(2019)], although in the former case a DCBH may still form. This suggests that, despite DCBHs need extremely peculiar conditions to form, they could explain at least part of the SMBH population at high redshift.

\section{Conclusions}

We have investigated the path to the formation of the early seeds of SMBHs. To address selfconsistently the formation of light and heavy BH seeds in primordial environments, we have developed and analysed numerical hydrodynamic simulations including the relevant physical and chemical processes taking place at early cosmological epochs: atomic and molecular non-equilibrium chemistry, cooling, star formation, feedback mechanisms, stellar evolution for popIII and popII-I generations, metal spreading from SNII, AGB and SNIa, as well as coupling with a multifrequency RT scheme according to adopted SEDs for popII-I and popIII radiative sources.

Light $\mathrm{BH}$ seeds originated during the final stages of standard stellar evolution can form within the first billion years with masses of the order of $\sim 1-10^{2} \mathrm{M}_{\odot}$. Their formation rate is tightly related to the SFR, although delayed by the mass-dependent stellar lifetimes of their progenitors (Fig. 3). The formation rate of light BH seeds at early times $(z \gtrsim 16)$ is initially dominated by popIII remnants for three main reasons: (i) popIII SF contribute significantly during the first bursts of cosmic star formation; (ii) popIII BHs have masses of the order of $10^{2} \mathrm{M}_{\odot}$, i.e. lifetimes of about $2 \mathrm{Myr}$, so their formation rate follows closely the SFR; (iii) popII-I BHs have masses of the order of 1-10 $\mathrm{M}_{\odot}$, 
i.e. lifetimes of at least $30 \mathrm{Myr}$, so their formation rate is delayed with respect to popIII ones. Later on, at redshift $z \lesssim 16$ popII-I remnants quickly take over, driven by the increasing popII-I SF, and their formation rate density becomes 2 dex larger then the popIII ones by $z \simeq 6$. Since by then light $\mathrm{BH}$ seeds cannot experience significant accretion, their role for SMBH formation is marginal.

Given the variety of configurations of their host candidates and the environmental features in which seed BHs might form, it is worth considering seeding mechanisms that require alternative scenarios rather than just focusing on star formation in massive halos [Buchner et al.(2019)]. In particular, heavy BH seeds could form through direct collapse of pristine gas in a single $\mathrm{BH}$ and give birth to a so-called direct-collapse BH, DCBH. After careful studies and within the limits of our simulations, we find that only powerful primordial stellar generations can determine the necessary conditions for DCBH formation, while standard stellar populations are too weak and, hence, unable to prevent molecule formation and fragmentation in the ambient gas. The DCBH host candidates feature gas masses of $1-3 \times 10^{5} \mathrm{M}_{\odot}$, turbulent regimes, pristine chemical composition and $\mathrm{H}_{2}$ dissociation due to $\mathrm{LW}$ radiation in the range $J_{\mathrm{LW}}=1-50 J_{21}$. They are found at a minimum distance of 5 physical $\mathrm{kpc}$ from the irradiating star forming site, being closer regions affected by photo-evaporation and metal enrichment that prevent gas direct collapse. Local non-linear processes, such as substructure formation and interactions, will influence DCBH evolution, as well. Thus, the necessary conditions required to form DCBHs must be complemented with information on the complex features of the local environment and a very fine balance between chemistry evolution and radiative transfer.

Although DCBHs might need extremely peculiar conditions, they could explain at least part of the SMBH population at high redshift. We note that here we strictly focus on the early phases of $\mathrm{BH}$ formation. The processes related to the following accretion and growth are complex issues that lie beyond the scope of this work. UV background distortions might be present and could be traced by metal ions [Graziani et al.(2019)], however their effects at such primordial times should have little impact on cosmic structure evolution. Alternative scenarios adopted for the cosmological model [Maio et al.(2006)], primordial non-gaussianities [Maio and Iannuzzi(2011), Maio(2011), Maio and Khochfar(2012)] or dark-matter nature [Maio and Viel(2015)] are unlikely to change substantially our conclusions on early BH seeds as derived from the properties of primordial cosmic star formation and metal enrichment [Maio et al.(2012)]. Primordial supersonic baryon streaming motions have the effect of delaying gas collapse, however, realistic values for supersonic streaming velocities would cause delays of some $10^{7} \mathrm{yr}$ [Maio et al.(2011)]. This corresponds to variations in $z$ of a few at very early epochs and smaller at lower $z$, therefore, primordial streaming motions will induce modest delays to $\mathrm{BH}$ seed formation, but they will not cause dramatic changes to the whole picture. The existence of tiny BHs formed during inflation has also been suggested, as well as BH formation from quarks or exotic dark matter. Given the large extent of such topics, we refer the interested reader to literature studies [Rubin et al.(2001), Khlopov et al.(2005), Khlopov(2010), Belotsky et al.(2019)].

In the future, observational synergies [Whalen et al.(2013), Koopmans et al.(2015), etc.] will shed light on primeval epochs giving information about the early state of neutral gas [Hutter et al.(2019)] and constraining the impacts of different radiative feedback models [Zackrisson et al.(2019)]. Combinations of optical, IR and X-ray surveys [Haiman et al.(2019)] will be promising for detecting early seed BHs and discerning between BH seeding channels using electromagnetic observations at redshifts $z>10$. 


\section{Acknowledgments}

We acknowledge detailed comments by the the referee, A. Dolgov, as well as useful discussions with D. Whalen, A. Santangelo and W. Kundt. This work has been supported through a research grant awarded to U.M. by the German Research Fundation (DFG) project n. 390015701 and the EU HPC-Europa3 Transnational Access Programme project n. HPC17ERW30. Numerical simulations and data analysis have been performed under the PRACE-2IP Programme, grant agreement $n$. RI-283493, and supported by the facilities of the Italian Computing Center (CINECA) and the Leibniz Institute for Astrophysics, Germany. We acknowledge the NASA Astrophysics Data System (ADS) and the JSTOR archive for their bibliographic tools.

\section{References}

[Rees(1984)] Rees, M. J. 1984. Black Hole Models for Active Galactic Nuclei. Annual Review of Astronomy and Astrophysics 22, 471.

[Dolgov and Silk(1993)] Dolgov, A., Silk, J. 1993. Baryon isocurvature fluctuations at small scales and baryonic dark matter. Physical Review D 47, 4244.

[Woosley and Weaver(1995)] Woosley, S. E., Weaver, T. A. 1995. The Evolution and Explosion of Massive Stars. II. Explosive Hydrodynamics and Nucleosynthesis. The Astrophysical Journal Supplement Series 101, 181.

[van den Hoek and Groenewegen(1997)] van den Hoek, L. B., Groenewegen, M. A. T. 1997. New theoretical yields of intermediate mass stars. Astronomy and Astrophysics Supplement Series 123, 305.

[Fan et al.(2001)] Fan, X., and 33 colleagues 2001. A Survey of z\&gt;5.8 Quasars in the Sloan Digital Sky Survey. I. Discovery of Three New Quasars and the Spatial Density of Luminous Quasars at z 6. The Astronomical Journal 122, 2833.

[Rubin et al.(2001)] Rubin, S. G., Sakharov, A. S., Khlopov, M. Y. 2001. The Formation of Primary Galactic Nuclei during Phase Transitions in the Early Universe. Soviet Journal of Experimental and Theoretical Physics 92, 921.

[Heger and Woosley(2002)] Heger, A., Woosley, S. E. 2002. The Nucleosynthetic Signature of Population III. The Astrophysical Journal 567, 532.

[Bromm and Loeb(2003)] Bromm, V., Loeb, A. 2003. Formation of the First Supermassive Black Holes. The Astrophysical Journal 596, 34.

[Thielemann et al.(2003)] Thielemann, F.-K., and 9 colleagues 2003. Nuclear cross sections, nuclear structure and stellar nucleosynthesis. Nuclear Physics A 718, 139.

[Khlopov et al.(2005)] Khlopov, M. Y., Rubin, S. G., Sakharov, A. S. 2005. Primordial structure of massive black hole clusters. Astroparticle Physics 23, 265.

[Springel(2005)] Springel, V. 2005. The cosmological simulation code GADGET-2. Monthly Notices of the Royal Astronomical Society 364, 1105.

[Begelman et al.(2006)] Begelman, M. C., Volonteri, M., Rees, M. J. 2006. Formation of supermassive black holes by direct collapse in pre-galactic haloes. Monthly Notices of the Royal Astronomical Society 370, 289. 
[Maio et al.(2006)] Maio, U., and 7 colleagues 2006. Early structure formation in quintessence models and its implications for cosmic reionization from first stars. Monthly Notices of the Royal Astronomical Society 373,869 .

[Maio et al.(2007)] Maio, U., Dolag, K., Ciardi, B., Tornatore, L. 2007. Metal and molecule cooling in simulations of structure formation. Monthly Notices of the Royal Astronomical Society 379, 963.

[Tornatore et al.(2007)] Tornatore, L., Borgan, S., Dolag, K., Matteucci, F. 2007. Chemical enrichment of galaxy clusters from hydrodynamical simulation. Monthly Notices of the Royal Astronomical Society 382,1050 .

[Khlopov(2010)] Khlopov, M. Y. 2010. Primordial black holes. Research in Astronomy and Astrophysics 10, 495.

[Maio et al.(2010)] Maio, U., Ciardi, B., Dolag, K., Tornatore, L., Khochfar, S. 2010. The transition from population III to population II-I star formation. Monthly Notices of the Royal Astronomical Society $407,1003$.

[Shang et al.(2010)] Shang, C., Bryan, G. L., Haiman, Z. 2010. Supermassive black hole formation by direct collapse: keeping protogalactic gas $\mathrm{H}_{2}$ free in dark matter haloes with virial temperatures $\mathrm{T}_{\text {vir }} \gtrsim 10^{4} \mathrm{~K}$. Monthly Notices of the Royal Astronomical Society 402, 1249.

[Campisi et al.(2011)] Campisi, M. A., Maio, U., Salvaterra, R., Ciardi, B. 2011. Population III stars and the long gamma-ray burst rate. Monthly Notices of the Royal Astronomical Society 416, 2760.

[Maio et al.(2011)] Maio, U., Koopmans, L. V. E., Ciardi, B. 2011. The impact of primordial supersonic flows on early structure formation, reionization and the lowest-mass dwarf galaxies. Monthly Notices of the Royal Astronomical Society 412, L40.

[Maio et al.(2011)] Maio, U., Khochfar, S., Johnson, J. L., Ciardi, B. 2011. The interplay between chemical and mechanical feedback from the first generation of stars. Monthly Notices of the Royal Astronomical Society 414, 1145.

[Maio and Iannuzzi(2011)] Maio, U., Iannuzzi, F. 2011. Baryon history and cosmic star formation in non-Gaussian cosmological models: numerical simulations. Monthly Notices of the Royal Astronomical Society 415, 3021.

[Maio(2011)] Maio, U. 2011. Gas distribution, metal enrichment and baryon fraction in Gaussian and non-Gaussian universes. Classical and Quantum Gravity 28, 225015.

[Mortlock et al.(2011)] Mortlock, D. J., and 16 colleagues 2011. A luminous quasar at a redshift of $\mathrm{z}=$ 7.085. Nature 474, 616.

[Wolcott-Green et al.(2011)] Wolcott-Green, J., Haiman, Z., Bryan, G. L. 2011. Photodissociation of $\mathrm{H}_{2}$ in protogalaxies: modelling self-shielding in three-dimensional simulations. Monthly Notices of the Royal Astronomical Society 418, 838.

[Maio and Khochfar(2012)] Maio, U., Khochfar, S. 2012. The imprint of cosmological non-Gaussianities on primordial structure formation. Monthly Notices of the Royal Astronomical Society 421, 1113.

[Maio et al.(2012)] Maio, U., Salvaterra, R., Moscardini, L., Ciardi, B. 2012. Counts of high-redshift GRBs as probes of primordial non-Gaussianities. Monthly Notices of the Royal Astronomical Society 426, 2078.

[Petkova and Maio(2012)] Petkova, M., Maio, U. 2012. Radiative feedback and cosmic molecular gas: numerical method. Monthly Notices of the Royal Astronomical Society 422, 3067. 
[Biffi and Maio(2013)] Biffi, V., Maio, U. 2013. Statistical properties of mass, star formation, chemical content and rotational patterns in early $\mathrm{z} \gtrsim 9$ structures. Monthly Notices of the Royal Astronomical Society 436, 1621.

[Dayal et al.(2013)] Dayal, P., Dunlop, J. S., Maio, U., Ciardi, B. 2013. Simulating the assembly of galaxies at redshifts z =6-12. Monthly Notices of the Royal Astronomical Society 434, 1486.

[Maio et al.(2013)] Maio, U., Ciardi, B., Müller, V. 2013. Simulating extremely metal-poor gas and DLA metal content at redshift z ? 7. Monthly Notices of the Royal Astronomical Society 435, 1443.

[Salvaterra et al.(2013)] Salvaterra, R., Maio, U., Ciardi, B., Campisi, M. A. 2013. Simulating high-z gamma-ray burst host galaxies. Monthly Notices of the Royal Astronomical Society 429, 2718.

[Whalen et al.(2013)] Whalen, D. J., and 7 colleagues 2013. Seeing the First Supernovae at the Edge of the Universe with JWST. The Astrophysical Journal 762, L6.

[Hirano et al.(2014)] Hirano, S., and 6 colleagues 2014. One Hundred First Stars: Protostellar Evolution and the Final Masses. The Astrophysical Journal 781, 60.

[Sugimura et al.(2014)] Sugimura, K., Omukai, K., Inoue, A. K. 2014. The critical radiation intensity for direct collapse black hole formation: dependence on the radiation spectral shape. Monthly Notices of the Royal Astronomical Society 445, 544.

[Koopmans et al.(2015)] Koopmans, L., and 43 colleagues 2015. The Cosmic Dawn and Epoch of Reionisation with SKA. Advancing Astrophysics with the Square Kilometre Array (AASKA14) 1.

[Maio and Tescari(2015)] Maio, U., Tescari, E. 2015. Origin of cosmic chemical abundances. Monthly Notices of the Royal Astronomical Society 453, 3798.

[Maio and Viel(2015)] Maio, U., Viel, M. 2015. The first billion years of a warm dark matter universe. Monthly Notices of the Royal Astronomical Society 446, 2760.

[Mancini et al.(2015)] Mancini, M., and 7 colleagues 2015. The dust mass in z \&gt; 6 normal star-forming galaxies.. Monthly Notices of the Royal Astronomical Society 451, L70.

[Habouzit et al.(2016)] Habouzit, M., Volonteri, M., Latif, M., Dubois, Y., Peirani, S. 2016. On the number density of 'direct collapse' black hole seeds. Monthly Notices of the Royal Astronomical Society 463, 529.

[Maio et al.(2016)] Maio, U., Petkova, M., De Lucia, G., Borgani, S. 2016. Radiative feedback and cosmic molecular gas: the role of different radiative sources. Monthly Notices of the Royal Astronomical Society 460, 3733 .

[Mancini et al.(2016)] Mancini, M., and 6 colleagues 2016. Interpreting the evolution of galaxy colours from $z=8$ to 5 . Monthly Notices of the Royal Astronomical Society 462, 3130.

[Johnson and Dijkstra(2017)] Johnson, J. L., Dijkstra, M. 2017. Enhanced direct collapse due to Lyman $\alpha$ feedback. Astronomy and Astrophysics 601, A138.

[Ma et al.(2017)] Ma, Q., Maio, U., Ciardi, B., Salvaterra, R. 2017. Constraining the PopIII IMF with high-z GRBs. Monthly Notices of the Royal Astronomical Society 466, 1140.

[Ma et al.(2017)] Ma, Q., Maio, U., Ciardi, B., Salvaterra, R. 2017. Metal enrichment signatures of the first stars on high-z DLAs. Monthly Notices of the Royal Astronomical Society 472, 3532.

[Bañados et al.(2018)] Bañados, E., and 17 colleagues 2018. An 800-million-solar-mass black hole in a significantly neutral Universe at a redshift of 7.5. Nature 553, 473. 
[Dolgov(2018)] Dolgov, A. D. 2018. Massive and supermassive black holes in the contemporary and early Universe and problems in cosmology and astrophysics. Physics Uspekhi 61, 115.

[Valiante et al.(2018)] Valiante, R., Schneider, R., Graziani, L., Zappacosta, L. 2018. Chasing the observational signatures of seed black holes at z \&gt; 7: candidate statistics. Monthly Notices of the Royal Astronomical Society 474, 3825.

[Belotsky et al.(2019)] Belotsky, K. M., and 9 colleagues 2019. Clusters of Primordial Black Holes. European Physical Journal C 79, 246.

[Haiman et al.(2019)] Haiman, Z., and 20 colleagues 2019. Electromagnetic Window into the Dawn of Black Holes. Bulletin of the American Astronomical Society 51, 557.

[Maio et al.(2019)] Maio, U., Borgani, S., Ciardi, B., Petkova, M. 2019. The seeds of supermassive black holes and the role of local radiation and metal spreading. Publications of the Astronomical Society of Australia 36, e020.

[Natarajan et al.(2019)] Natarajan, P., and 16 colleagues 2019. Disentangling nature from nurture: tracing the origin of seed black holes. Bulletin of the American Astronomical Society 51, 73.

[Graziani et al.(2019)] Graziani, L., Maselli, A., Maio, U. 2019. UV background fluctuations traced by metal ions at $\mathrm{z} \approx 3$. Monthly Notices of the Royal Astronomical Society 482, L112.

[Hutter et al.(2019)] Hutter, A., and 28 colleagues 2019. A proposal to exploit galaxy-21cm synergies to shed light on the Epoch of Reionization. Bulletin of the American Astronomical Society 51, 57.

[Buchner et al.(2019)] Buchner, J., Treister, E., Bauer, F. E., Sartori, L. F., Schawinski, K. 2019. On the Prevalence of Supermassive Black Holes over Cosmic Time. The Astrophysical Journal 874, 117.

[Zackrisson et al.(2019)] Zackrisson, E., and 18 colleagues 2019. Bubble mapping with the Square Kilometer Array - I. Detecting galaxies with Euclid, JWST, WFIRST and ELT within ionized bubbles in the intergalactic medium at z\&gt;6. arXiv e-prints arXiv:1905.00437.

\section{DISCUSSION}

ANDREA SANTANGELO: How the scenario for SMBH formation would change if predictions about the existence of primordial BH is taken into account?

ANSWER: small primordial BHs should merge to form larger BHs, however they might not have enough time to coalesce and grow to the desired masses by redshift $z \sim 7$. Mechanisms of very massive (even supermassive) primordial BH formation in the very early universe have been proposed [Dolgov and Silk(1993), Dolgov(2018)], but this is still a debated issue.

WOLFGANG KUNDT: You treat your SMBHs as stationary objects. Why are you permitted to ignore their rapid growth via accretion?

ANSWER: We strictly focus on the early phases of SMBH formation. The processes related to the following accretion and growth episodes are complex issues that lie beyond the scope of this work. 
DAN WHALEN: Nice talk! My question is: What do you think about the relative roles of LW backgrounds and supersonic baryon streaming motions in the primordial Universe in suppressing popIII star formation in haloes until they grow to $10^{7}-10^{8} \mathrm{M}_{\odot}$ ?

ANSWER: LW backgrounds and supersonic baryon streaming motions have both the effect of delaying gas collapse and an exact assessment will depend on the precise values adopted for baryon streaming velocities and LW fluxes. Realistic values for supersonic baryon streaming velocities should imply shorter delays than the ones expected from large LW fluxes. 